\title{
Barometric and magnetic observations of vertical acoustic resonance and resultant generation of field-aligned current associated with earthquakes
}

\author{
Toshihiko Iyemori ${ }^{1}$, Yoshikazu Tanaka ${ }^{2}$, Yoko Odagi ${ }^{1}$, Yasuharu Sano ${ }^{3}$, Masahiko Takeda $^{1}$, Masahito Nose ${ }^{1}$, \\ Mitsuru Utsugi ${ }^{1}$, Domingo Rosales ${ }^{4}$, Edwin Choque ${ }^{4}$, Jose Ishitsuka ${ }^{4}$, Sadato Yamanaka ${ }^{1}$, \\ Kunihito Nakanishi ${ }^{1}$, Mitsuru Matsumura ${ }^{5}$, and Hiroyuki Shinagawa ${ }^{6}$ \\ ${ }^{1}$ Graduate School of Science, Kyoto University, Kyoto 606-8502, Japan \\ ${ }^{2}$ Setsunan University, Neyagawa 572-8503, Japan \\ ${ }^{3}$ Asahi University, Mizuho 501-0223, Japan \\ ${ }^{4}$ Instituto Geofisico del Peru, Lima 3, Peru \\ ${ }^{5}$ University of Electro Communications, Chofu 182-8585, Japan \\ ${ }^{6}$ National Institute of Information and Communications Technology, Koganei 184-8795, Japan
}

(Received October 2, 2012; Revised January 9, 2013; Accepted February 8, 2013; Online published September 17, 2013)

\begin{abstract}
Three rare occasions are introduced, where the excitation of vertical acoustic resonance between the ground and the ionosphere, and the resultant generation of a field-aligned current, just after earthquakes are observationally confirmed. In the case of two inland earthquakes, barometric observations very close to the epicenters (i.e., only $30 \mathrm{~km}$ apart) were available, and they showed a sharp spectral peak which appeared within one hour after the origin time and lasted a few hours. The observed periods of the spectral peaks around 260 seconds are close to the period of the theoretically-expected fundamental mode of the resonance. On the other hand, magnetic observations on the ground showed a dominant period at 220-230 seconds which corresponds to the first overtone among theoretically-expected major resonance peaks. In the third case, i.e., during the 2010 Chile earthquake, a long-period magnetic oscillation in the east-west direction, which has two major resonance periods at 265 and 190-195 seconds, was observed on the night-side magnetic dip equator in Peru, where the distance is more than $2600 \mathrm{~km}$ from the epicenter, under a very quiet geomagnetic condition. The oscillation was interpreted as the effect of field-aligned current generated through a dynamo process in the ionosphere over the epicenter caused by the resonance.
\end{abstract}

Key words: Acoustic wave, field-aligned current, earthquake, resonance, ionospheric dynamo.

\section{Introduction}

During the 2004 Sumatra earthquake, a peculiar Pc5 magnetic pulsation and GPS-TEC pulsation were observed about 12 minutes after the origin time in the north-east part of Thailand, Phimai, where the distance from the epicenter was about $1500 \mathrm{~km}$ (Iyemori et al., 2005; Choosakul et al., 2009). Because both phenomena have a sharp spectral peak at about 4 minutes (to be exact, 3.6 minutes), they were explained as the results of vertical acoustic resonance between the ocean surface and the ionosphere, excited by the sudden vertical movement of the ocean surface at the epicenter. In fact, a similar oscillation in the GPS-TEC data has been observed during the 2011 Off the Pacific Tohoku Earthquake (Saito et al., 2011; Tsugawa et al., 2011), and a numerical simulation study on atmospheric gravity waves, including the acoustic mode, strongly supports the excitation of vertical acoustic resonance (see Matsumura et al., 2011).

Although vertical acoustic resonance is the most plausible mechanism of the 4-minute oscillation observed after the earthquakes, there has been no direct evidence of

Copyright (C) The Society of Geomagnetism and Earth, Planetary and Space Sciences (SGEPSS); The Seismological Society of Japan; The Volcanological Society of Japan; The Geodetic Society of Japan; The Japanese Society for Planetary Sciences; TERRAPUB.

doi:10.5047/eps.2013.02.002 acoustic resonance by atmospheric pressure observations. As for the case of volcanic eruption, there exist some reports on atmospheric pressure oscillation with a long period. During the $1991 \mathrm{Mt}$. Pinatubo eruption, a long-period seismic wave having a period about 4 minutes (to be exact, 3.7 minutes) was observed worldwide, and the phenomenon was explained as the effect of vertical acoustic resonance (Kanamori and Mori, 1992; Kanamori et al., 1994). However, there was no digital recording of atmospheric pressure near the volcano available for spectral analysis.

In this paper, we show the barometric data obtained at the sites close to the epicenter of the two inland type (i.e., caused by movement of an active fault) earthquakes that occurred in Japan, and compare their spectra with those of geomagnetic observations. The comparison strongly supports the excitation of vertical acoustic resonance over the epicenter just after the earthquakes, and the generation of electric currents in the ionosphere.

Evidence of the generation of a field-aligned current associated with acoustic resonance is also shown from magnetic observations in Peru during the 2010 Chile earthquake. 


\section{Method to Identify the Vertical Acoustic Reso- nance}

After the 2004 Sumatra earthquake, we began microbarometric observations at stations in Japan and outside of Japan. The pressure sensor used is the Vaissala PTB210 and the 1-second-averaged data from 490 samples/s A/D output have been recorded. The actual resolution of the pressure variation is about 1 Pascal.

In general, the pressure variation caused by the acoustic mode atmospheric waves is smaller than that of internal gravity waves having a longer period (i.e., typically more than 6 or 7 minutes) in the lower atmosphere, and it is not easy to find out from the time series plot of the pressure data of our observations because of the limitation of resolution and low $\mathrm{S} / \mathrm{N}$ ratio. However, it is possible to detect by spectral analysis with high-frequency (period) resolution, because the vertical acoustic resonance has 3 major resonance peaks theoretically well defined (calculated) with a realistic atmospheric model. That is, it is well established by several authors that the major 3 resonant peaks exist at around 260270 seconds, $220-230$ seconds, and 190-200 seconds (e.g., Tahira, 1995; Lognonne et al., 1998; Nishida et al., 2000; Shinagawa et al., 2007) although the period may vary for some extent depending on the atmospheric model, boundary condition, etc. If one or more spectral peaks close to the expected period of resonance appear just after the origin time of an earthquake with an amplitude larger than that of the normal condition or that before the earthquake, we may conclude with a high probability that this indicates the excitation of the resonance by the earthquake. To analyze this with high-frequency resolution, we adopt the MEM (Maximum Enthoropy Method) in this paper.

From the numerical simulation study by, for example, Shinagawa et al. (2007) or Matsumura et al. (2011), the region of vertical acoustic resonance in the initial stage is limited to the area over the epicenter, and it is expected that the barometric variation associated with the resonance can be observed in the area close to the epicenter, say within $100 \mathrm{~km}$ depending on the scale of the earthquake. On the other hand, the ionospheric current effect, i.e., the magnetic variation, may be observed over a wider area because the electric current circuit can extend outside the resonance region by the return current and/or the field-aligned current, as discussed in a later section.

After we started barometric observation in 2006, two inland-type earthquakes occurred with rather a large magnitude near the barometric observation sites, and the distance between the epicenters and the observation site was only 30 $\mathrm{km}$ or less. Because of the sparse barometric observation sites, these two cases are very rare events.

In the paper by Iyemori et al. (2005), an ionospheric dynamo process, and associated field-aligned current generation by the resonance, were proposed, and the Pc5 magnetic pulsation observed in Thailand just after the Sumatra earthquake was explained as the effect of an ionospheric current system with a field-aligned current. Since the conductivity parallel to the magnetic field is very high, the polarization electric field is easily mapped to the conjugate points, and thus a part of the return current flows as the field-aligned current and closes through the opposite hemisphere. The same process is expected to occur in general, including the case of inland-type earthquakes. Therefore, we also analyze geomagnetic data observed on the ground. To show the existence of a field-aligned current generated by the dynamo process, an event associated with the 2010 Chile earthquake which occurred off the coast of central Chile on February 27,2010 , at 06:34 UTC, having a magnitude of 8.8 on the moment magnitude scale, is also introduced.

\section{Analysis}

Figure 1 shows the epicenters of two inland earthquakes and the location of barometric and geomagnetic observation sites used in this paper. In this section, we describe the observational results found in the three cases.

\subsection{Iwate-Miyagi Nairiku earthquake}

An inland earthquake with magnitude 7.2 occurred on June 13, 2008, 23:43 UT. The epicenter was at (39.0N, 140.9E) and the depth was $8 \mathrm{~km}$. As shown in Fig. 1, the epicenter was close to the NAO/Mizusawa (MIZ) observatory at $(39.11 \mathrm{~N}, 141.20 \mathrm{E})$ where a superconducting gravimeter was running. The distance between the epicenter and the site MIZ is only about $30 \mathrm{~km}$. The gravity measurement itself was stopped by the earthquake; however, a barometer attached to the gravimeter continued the measurement. It had a similar sensitivity as our barometric observations at several sites, including Shigaraki used in this paper. In this section, we analyze the MIZ barometric data and geomagnetic data obtained at Memambetsu (MMB) at (43.91N, 144.19E) and the Kakioka (KAK) magnetic observatory at $(36.23 \mathrm{~N}, 140.19 \mathrm{E})$. The time resolution of these data isone second, and they are high-pass filtered with a cutoff period around 10 minutes, which is not very sharp because of the Gaussian type filter. After the filtering, a MEM (Maximum Entropy Method) with a lag of 1200 seconds is applied for each 1-hour interval, shifting 30 minutes, to get the power spectral density (PSD) with a high frequency (period) resolution to identify the resonance period. The PSD is calculated at every period with a 5 -second interval. The

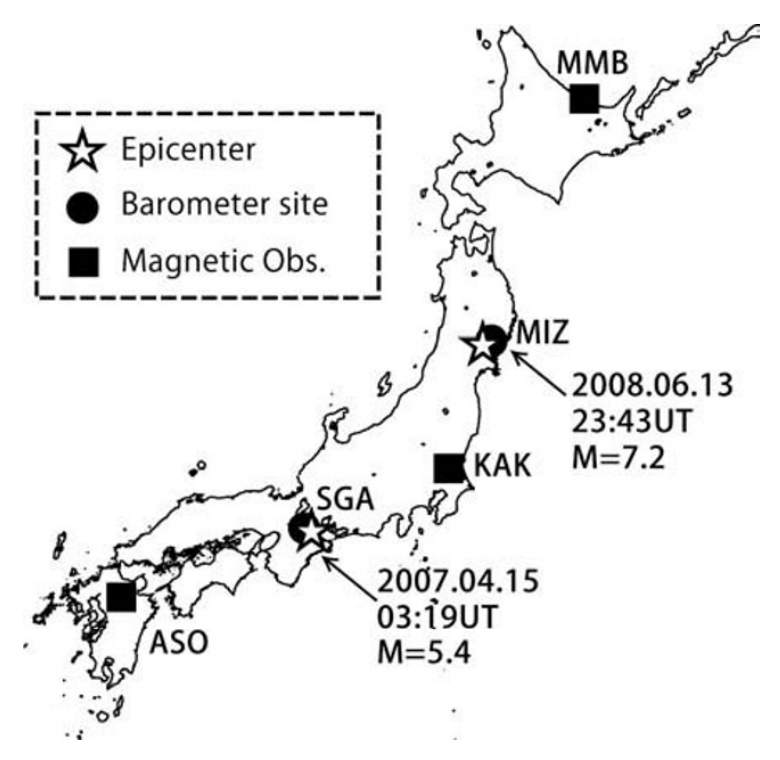

Fig. 1. Location of the epicenters and observation sites for the two inland earthquakes analyzed in this paper. 


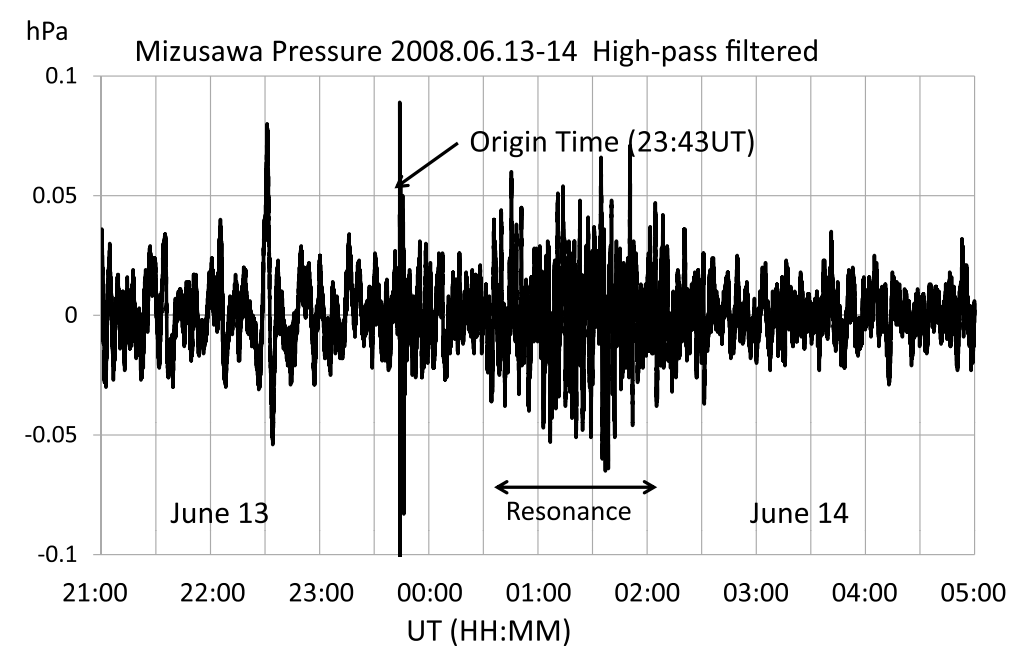

Fig. 2. Barometric data obtained at Mizusawa observatory, which is close to the epicenter of the 2008 Iwate-Miyagi Nairiku earthquake. Immediately after the origin time, a spike is seen, which may be an effect of seismic displacement. The amplitude of the barometric oscillation for 00:30-02:30 UT is larger than that before the origin time, and a resonance peak appears in the PSD during the interval.

same filter and MEM parameters are applied for other data used in this paper.

As seen in Fig. 2, the amplitude of the barometric oscillation increases about 40 minutes after the origin time at 23:43 UT and it lasts about two hours. Immediately after the origin time a large spike is seen, which may indicate the arrival of the seismic waves and ground movement in the vertical direction. For the interval of 01:00-02:00 UT, a clear spectral peak at a period of 260 seconds appears as shown in Fig. 3, which is close to the theoretically predicted fundamental mode of the vertical acoustic resonance. Such a peak did not exist just before the earthquake. By an examination of the PSD for the periods shifted every 30 minutes, this sharp spectral peak appears for the period 00:30-02:00 UT as indicated by the green and red lines.

Figures 4 and 5 show the PSD of the geomagnetic fields obtained at two geomagnetic observatories-Memambetsu and Kakioka-both of which are about $300 \mathrm{~km}$ from the epicenter. The geomagnetic activity was very low for several hours before, and after, the origin time of the earthquake. For example, the geomagnetic $K_{\mathrm{p}}$ index was less than 1 during the interval (e.g., http://isgi.cetp.ipsl.fr/). Although they do not show a sharp peak with the same period of 260 seconds observed by the barometer at Mizusawa, they show several peaks that are close to the periods theoretically predicted as the major acoustic resonance modes, including a peak at $265 \mathrm{~s}$ which is close to $260 \mathrm{~s}$. That is, at Memambetsu, the peaks around 265 (275), 220 and 185 seconds appear after the earthquake. At Kakioka, peaks at 220 (225) and 180 seconds appear.

The peaks at 265 and 275 seconds in the magnetic data may also be the fundamental resonance period. However, they slightly differ from that of the pressure data, i.e., 260 seconds. This difference suggests that the location of the resonance which caused the magnetic variation at Memambetsu was different from that of the pressure data observed near the epicenter. This point is mentioned again in the Discussion section.

\subsection{Mieken-Chubu earthquake}

An inland earthquake with magnitude 5.4 occurred at 03:19 UT on April 15, 2007. The epicenter was at $(34.8 \mathrm{~N}$, $136.4 \mathrm{E})$ and the depth was $10 \mathrm{~km}$. One of our barometric observation sites, Shigaraki (SGA) at $(34.85 \mathrm{~N}, 136.11 \mathrm{E})$, was very close to this epicenter. The distance between the epicenter and the site SGA was only about $30 \mathrm{~km}$.

Figure 6 shows a time series plot of high-pass filtered barometric data. Because of the local turbulence, it is not very easy to recognize the effect of the acoustic resonance from this line plot. However, looking at it carefully, the amplitude of oscillation becomes larger from around 03:30 UT.

If we take the power spectra of the barometric data shown in Fig. 6, the resonance peaks clearly appear. Figure 7 shows the PSD for the period before, and after, the origin time. Each spectrum was taken for each one-hour period. A clear peak with a period of 250 seconds appears for the period 03:30-04:30 UT when the amplitude in Fig. 6 slightly increases, which indicates the excitation of the fundamental mode of acoustic resonance, and the peak seems to shift to $265 \mathrm{~s}$ for the next interval, 04:00-05:00 UT.

The PSD of geomagnetic variation observed at the Aso (ASO) station $(32.88 \mathrm{~N}, 131.01 \mathrm{E})$ is shown in Fig. 8. Although not shown, the Kakioka data shows a similar spectra with a smaller amplitude. The geomagnetic activity for several hours before, and after, the earthquake was moderately quiet; for example, the $K_{\mathrm{p}}$ index was 2 during the interval. A small spectral peak at $250 \mathrm{~s}$ appears in the $D$-component, which may correspond to the sharp peak of the barometric spectrum in Fig. 7; however, larger peaks that fit better with the theoretically expected resonance peaks appear at $275 \mathrm{~s}$ and $230 \mathrm{~s}$ for 03:00-04:00 UT.

\subsection{Chile earthquake}

The 2010 Chile earthquake occurred off the coast of central Chile on February 27, 2010, at 06:34 UT (03:34 LT), having a magnitude of 8.8 . The epicenter was at $35.9 \mathrm{~S}$, $72.7 \mathrm{~W})$. About 15 minutes after the origin time, a longperiod geomagnetic pulsation having a period of about 4 


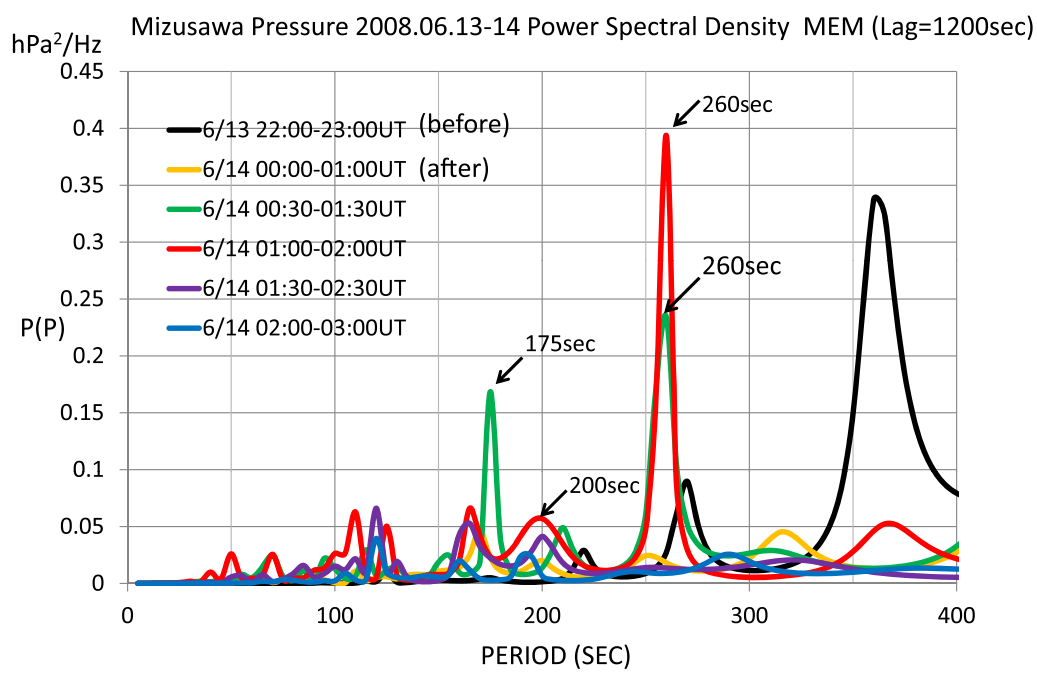

Fig. 3. Power spectral density of the barometric data obtained at Mizusawa before (black line) and after the origin time. A clear spectral peak at 260 $\mathrm{s}$ appears for one or two hours after the origin time (green and red lines), which indicates the excitation of vertical acoustic resonance caused by the earthquake.

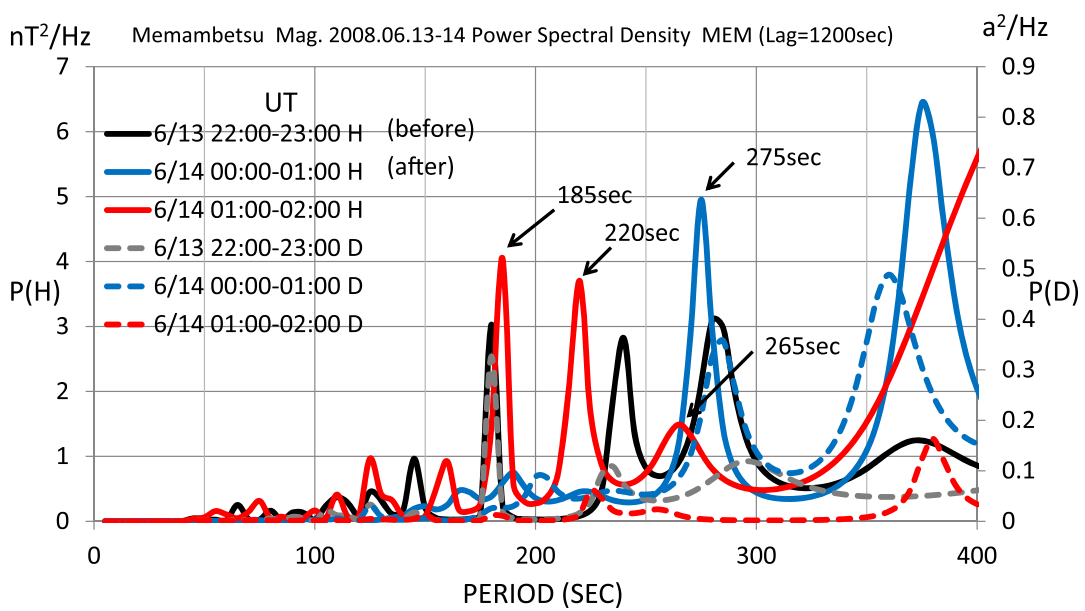

Fig. 4. Power spectral density of geomagnetic variation observed at Memambetsu observatory. Spectral peaks for 01:00-02:00 UT (red solid line) shows the three theoretically expected major peaks, i.e., at $265 \mathrm{~s}, 220 \mathrm{~s}$ and $185 \mathrm{~s}$. The black lines indicate the PSD before the earthquake. Note that the unit of the magnetic $D$ component is in "minute" of angle and indicated as "a" for simplicity in the figure.

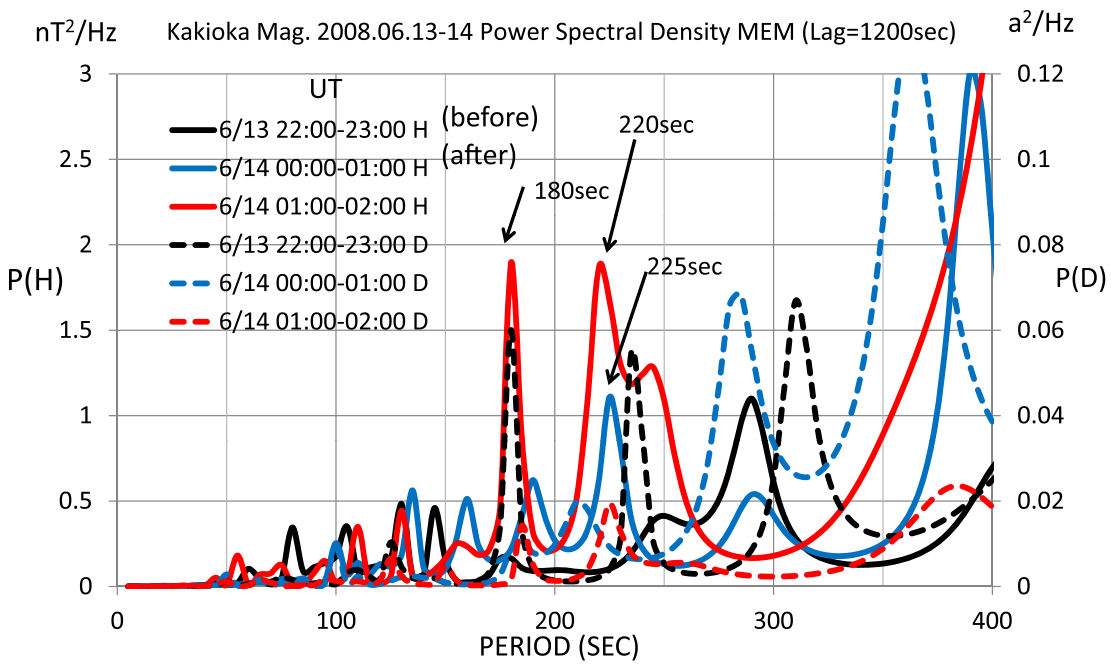

Fig. 5. Power spectral density of geomagnetic variation observed at Kakioka observatory. Spectral peaks for 01:00-02:00 UT (red solid line) include the peak at $220 \mathrm{~s}$ theoretically expected as the first overtone. 


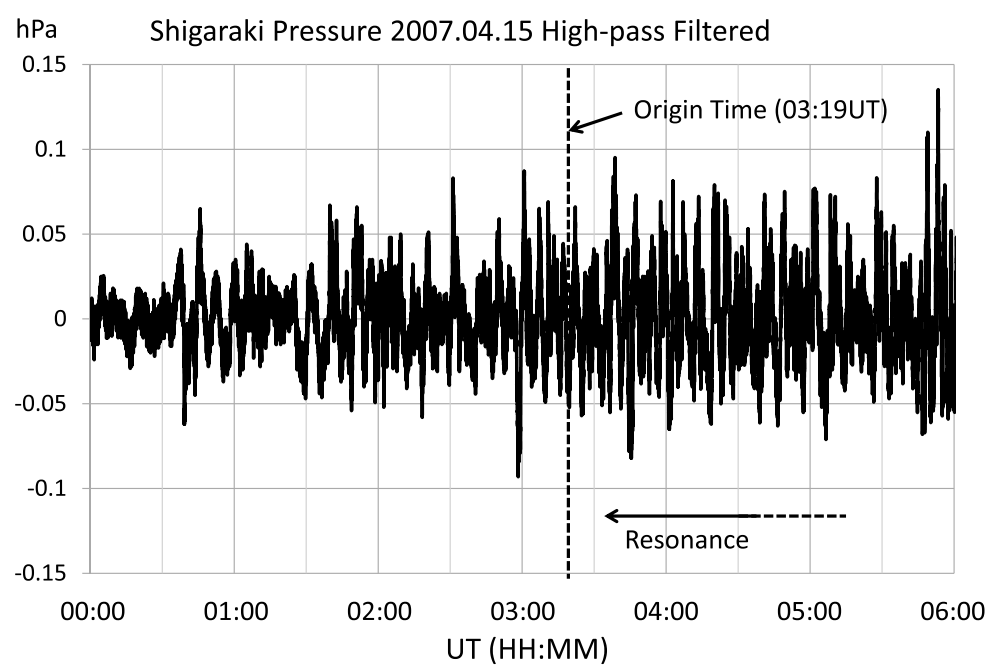

Fig. 6. Barometric variation observed at the Shigaraki station around the Mieken-Chubu earthquake.

minutes was observed at two magnetic observatories on the geomagnetic dip equator: Huancayo at $(12.04 \mathrm{~S}, 75.32 \mathrm{~W})$ and Ancon at $(11.77 \mathrm{~S}, 77.15 \mathrm{~W})$ in Peru. The distance between the epicenter and these observatories is about 2650 km. Figures 9(a) and 9(b) respectively show magnetic fluctuations observed at Huancayoand Ancon. The data from Huancayo are one-minute averages and those from Ancon have a one-second resolution. Note that the unit of the $D$ component at Ancon is expressed in nT, i.e., an orthogonal component to the $H$-component on the horizontal plane. The seismic waves arrived at Huancayo around 06:40 UT, 8 minutes earlier than the start of the magnetic variation. The arrival of the seismic wave was detected as the effect of a sensor oscillation of a suspended type magnetometer, which is not used in this paper.

One remarkable characteristic is that the oscillation is observed only in the $D$ (declination) component, i.e., in the East-West direction at Huancayo, although a smaller oscillation is seen also in the $Z$ (vertical) component at Ancon. Because of the local time before dawn, under very quiet geomagnetic activity as seen in the $\mathrm{AE}$ indices, i.e., hourly $\mathrm{AE}$ index values of about $30 \mathrm{nT}$ or less (see http://wdc.kugi.kyoto-u.ac.jp/dstae/index.html) with the lowest $K_{\mathrm{p}}$ index, $0_{0}$, and under a rather stable solar wind condition, i.e., a solar wind speed around $320 \mathrm{~km} / \mathrm{s}$ and a density around 4.5 protons $/ \mathrm{cm}^{3}$ (see http://omniweb.gsfc.nasa.gov/form/omni_min.html), the possibility of a magnetospheric disturbance, such as a substorm onset or direct solar wind disturbance effect, is very low. It is also not plausible that the oscillation comes from the sensor oscillation caused by low-frequency seismic waves, such as the Rayleigh or Love waves, because of the much longer period (i.e. about 4 minutes) than that of the Rayleigh or Love waves (i.e., their major period is normally shorter than 30 seconds or at most 1 minute or so-e.g. Selby et al., 2005). This is also confirmed, for example, by a seismometer record at Tungurahua, Ecuador.

Figure 10 shows the PSD of the geomagnetic $D$ component observed at Ancon. The spectrum clearly indicates two peaks at $265 \mathrm{~s}$ and $190-195 \mathrm{~s}$ that exactly correspond to the fundamental and the second overtone of the resonance frequency, respectively. The only plausible explanation of this magnetic oscillation in the east-west direction is the effect of the field-aligned current generated by the acoustic resonance over the epicenter through the ionospheric dynamo process, which flows over the magnetic equator as schematically depicted in Fig. 11(a). This is because: (1) the oscillation is mainly in the east-west component; (2) the spectral peaks correspond to the resonance frequencies; and (3) various conditions such as the solar wind parameters, the local time and geomagnetic disturbance, do not fit the condition suitable for the generation of the known geomagnetic pulsations of a space plasma origin.

\section{Summary and Discussion}

The emergence of clear spectral peaks both in the barometric data near the epicenter, and in the geomagnetic data, strongly supports the causal relationship between the earthquake and the excitation of the vertical acoustic resonance near the epicenter. The peaks have a period close to the major modes of the resonance that has been predicted theoretically (e.g., Tahira, 1995; Lognonne et al., 1998; Nishida et al., 2000; Shinagawa et al., 2007), and their magnetic effects, through the ionospheric dynamo process, have also been expected since 2004 Sumatra earthquake (Iyemori et al., 2005).

As for the cases of the two inland earthquakes introduced in this paper, the sharp spectral peaks in the barometric data have a period close to the fundamental mode (i.e., at $265 \mathrm{~s}$ and $250 \mathrm{~s}$, respectively), although the main spectral peaks in the geomagnetic data have periods that are close to other resonance periods around 220-230 s (i.e., the first overtone) or $190 \mathrm{~s}$ (i.e., the second overtone) rather than that of the fundamental mode. This tendency was also observed during the 2004 Sumatra earthquake, where the first overtone (3.6 minutes) dominated in the magnetic data during the initial one or two hours. The spectral peaks of the barometric variation at the 2008 Iwate-Miyagi Nairiku earthquake show the first overtone (at $210 \mathrm{~s}$ ) and the second overtone (at $175 \mathrm{~s}$ ) during the interval which is 30 minutes 


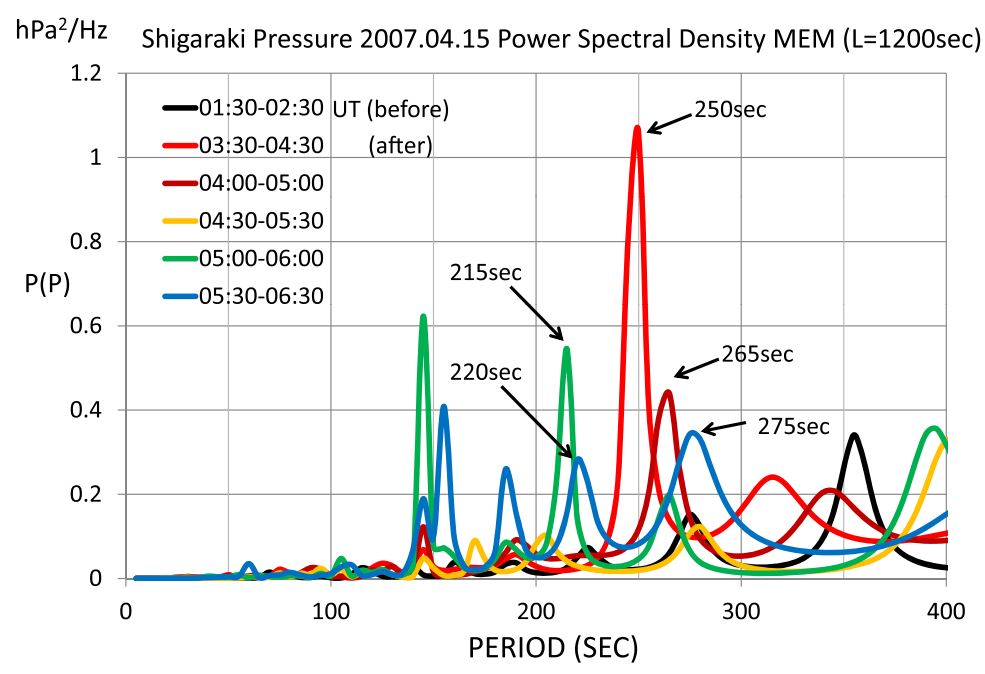

Fig. 7. Power spectral density of the barometric variation before, and after, the origin time at 03:19 UT on April 15, 2007. Just after the origin time, 03:30-04:30 UT, a sharp peak at period $250 \mathrm{~s}$ appears, which is close to, or slightly shorter than, the fundamental mode of the vertical acoustic resonance, and the peak seems to shift to 265 and 275 seconds with time.

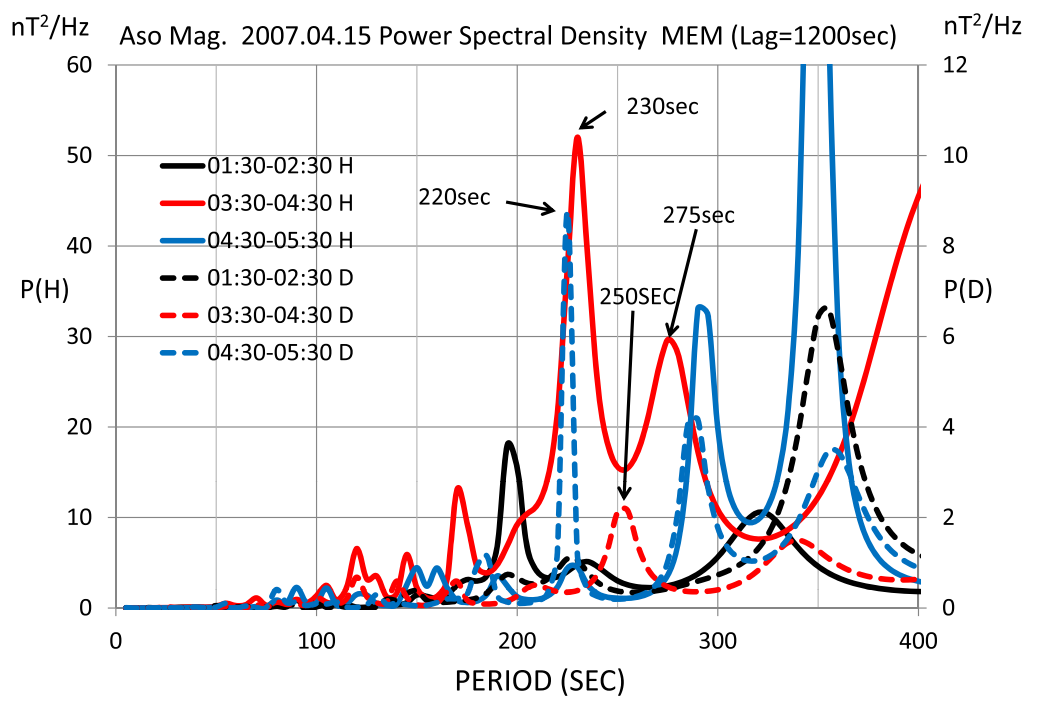

Fig. 8. Power spectral density of the magnetic variation at Aso station. The spectra of the Horizontal $(H)$ and Declination $(D)$ components are shown. Two peaks in the $H$ component at $275 \mathrm{~s}$ and $230 \mathrm{~s}$ appear just after the origin time, which correspond to the fundamental, and the first overtone, of the resonance modes, respectively. A small peak at $250 \mathrm{~s}$ also appears in the $D$ component, which may correspond to the peak seen in the barometric data obtained at Shigaraki.

earlier than the period when the highest peak appears in Fig. 3 (green line), and these peaks are consistent with the magnetic signature indicated in Figs. 4 and 5 although there exists a small (i.e., about 10 seconds) difference in the period.

The periods of the resonance peaks of the magnetic field variation are not necessarily the same with those of the pressure variation, as seen in the case of the 2008 Iwate-Miyagi Nairiku earthquake. That is, as seen in Fig. 3, the pressure data shows the fundamental mode with a period of $260 \mathrm{sec}-$ onds. On the other hand, the Memambetsu geomagnetic data show the spectral peaks at a period of 275 or $265 \mathrm{sec}-$ onds. The difference suggests that the resonance occurs not only over the epicenter, but also at a different location with a slightly different resonance period as the seismic wave, or the atmospheric gravity waves, propagate from the epicen- ter. To investigate the cause of the difference, we need a collocated observation of magnetic field and pressure variation as well as multi-points observation.

On the other hand, the 2010 Chile earthquake occurredbefore dawn and the PSD of the geomagnetic oscillation observed in Peru (HUA and ANC) shows spectral peaks at $265 \mathrm{~s}$ and $190-195 \mathrm{~s}$ and no peak at 220-230 s. This difference could be explained as the altitude difference of the dynamo layer between the dayside and nightside. That is, the mode having a large amplitude at the ionospheric dynamo layer, which is around $110-120 \mathrm{~km}$ on the dayside and a slightly higher altitude on the nightside, could dominate. To confirm this, and also to solve the problem of the small difference in the period mentioned in the previous paragraph, the height dependence of resonance oscillation and horizontal movement of the resonance region (or hor- 
(a)

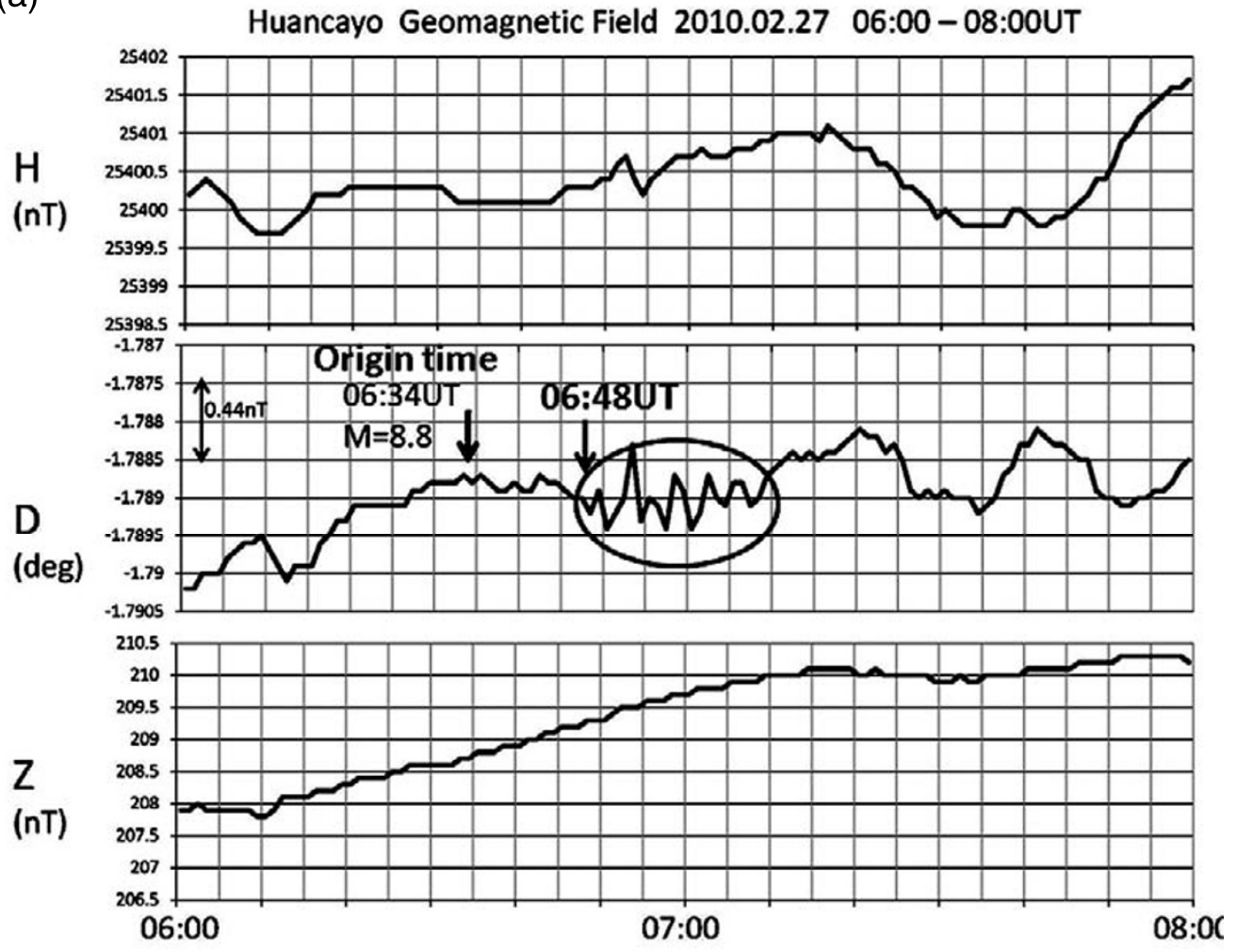

(b)
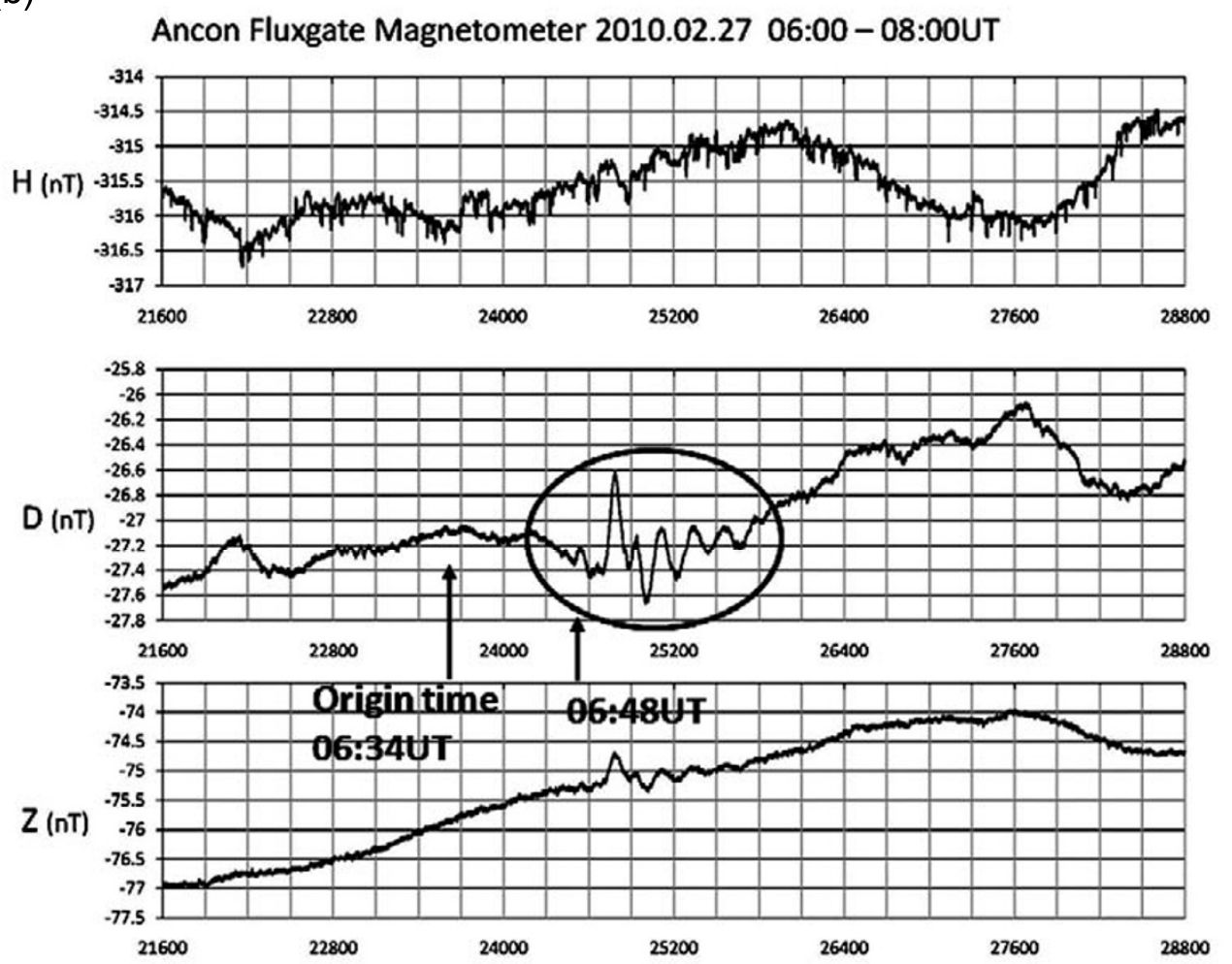

Fig. 9. (a) Geomagnetic variation observed at Huancayo, Peru just after the origin time of the 2010 Chile earthquake. Oscillation is observed only in the declination (i.e., East-West component) which suggests the effect from a trans-equatorial field-aligned current generated in the ionosphere over the epicenter. The field-aligned current closes by the ionospheric current in the opposite hemisphere as depicted in Fig. 11(a). The arrival of the seismic wave was 8 minutes earlier (around 06:40 UT) than the onset of magnetic oscillation at 06:48 UT. (b) One second resolution geomagnetic data from Ancon observatory in Peru on the magnetic dip equator. The magnetic oscillation appears mainly in the $D$ component, although a smaller variation is also seen in the $Z$ (vertical) component. The small spiky variations in the $H$ (horizontal) component are due to artificial noise caused by incomplete contact of the sensor cable. Note that the $D$ component is expressed in units of nT. 


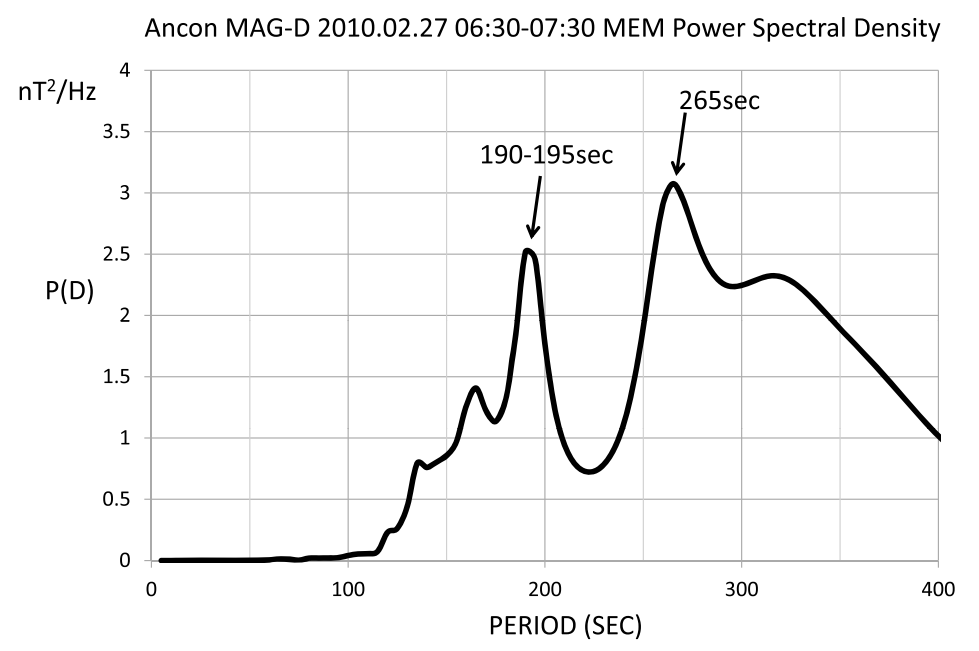

Fig. 10. Power spectral density of the magnetic $D$-component obtained at the Ancon observatory. Two spectral peaks at $265 \mathrm{~s}$ and $190-195 \mathrm{~s}$ correspond to the fundamental, and the second overtone, of the major resonance peaks.

\section{(a) Closure by Pedersen current}

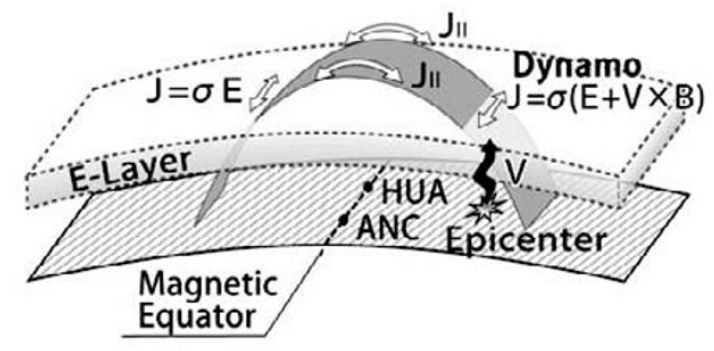

\section{(b) Closure by Hall current}

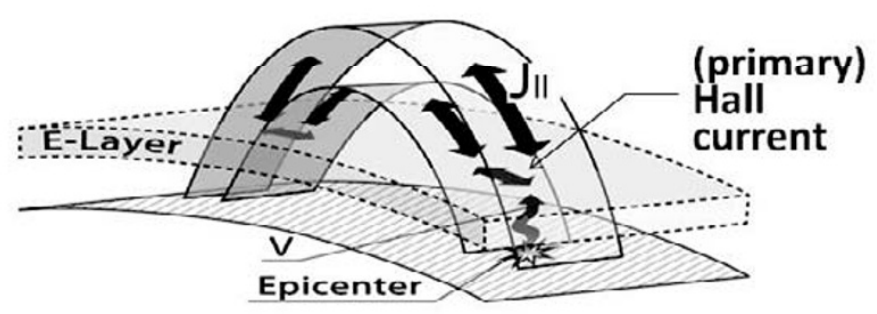

Fig. 11. Possible current closure through field-aligned current. The atmospheric motion $(V)$ as the dynamo driver is assumed to be in the vertical direction for simplicity. The secondary Hall current is not depicted.

izontal propagation of the acoustic/internal gravity waves) should be simulated with a realistic model.

Figure 11 presents schematic drawings of the closure of the dynamo current through the field-aligned current. Figure 11(a) shows the closure with the Pedersen current in the ionosphere. Here, we assume that the motion of the neutral atmosphere is in the vertical direction; therefore, the dynamo electric field is mainly in the east-west direction. Because the velocity of atmospheric gravity waves is much slower than that of Alfvén waves, the dynamo region should be bounded within a few hundred $\mathrm{km}$. That is, in a timescale about 10 minutes, i.e., the timescale for the acoustic gravity wave to reach the ionospheric height and return back to the ground, the waves can spread horizontally at most a few hundred kilometers. At the boundary, the electric charge accumulates and the ionospheric current diverts along the geomagnetic field line forming a polarized electric field. This case may be more suitable on the nightside, where the Pedersen conductivity is relatively larger than the Hall conductivity. Figure 11(b) shows the closure with the Hall current and may correspond to the case on the dayside, where the Hall conductivity is larger than the Pedersen conductivity.

For the 2010 Chile earthquake, the closure depicted in Fig. 11(a) may be the case because it happened on the nightside. The tracing of geomagnetic lines of force with the IGRF model indicates that the geomagnetic line of force from $130 \mathrm{~km}$ above the epicenter passes the longitude and altitude at $71.5 \mathrm{~W}$ and $1132 \mathrm{~km}$, respectively, on the mag- 
netic dip equator. That is, it is 3.8 degrees east of Huancayo and 5.7 degrees east of Ancon. Taking into account the resonance region with a diameter of about a few hundred $\mathrm{km}$, the Huancayo observatory could be under the west side field-aligned current depicted in Fig. 11(a), where no vertical magnetic effect appears. On the other hand, the Ancon observatory could be on the west of the west side fieldaligned current, and, hence, it could have a small oscillation also in the vertical component. The phase relation between the $D$ (eastward positive) and the $Z$ (downward positive) of the variation at Ancon shown in Fig. 9(b) has a positive and linear correlation, which is consistent with the above explanation.

For the two inland earthquakes, the closure depicted in Fig. 11(b) may be the case, because they occurred on the dayside where the Hall conductivity is larger than the Pedersen conductivity. On the dayside, the ionospheric Hall current which circulates in the ionosphere mainly contributes to the magnetic variation on the ground (e.g., Tamao, 1986; Fujita and Tamao, 1988). For the current closure of the Fig. 11(b) type, the primary Hall current in the north-south direction closes the current circuit through the field-aligned current. On the other hand, the Hall current which closes in the ionosphere flows mainly in the east-west direction by the polarized electric field in the north-south direction. This polarized electric field is caused by the primary Hall current in the north-south direction driven by the dynamo electric field in the east-west direction, because the dynamo region is limited both in latitude and longitude and the magnetically-conjugate ionosphere in another hemisphere has a finite resistivity. This polarized electric field drives the secondary Hall current in the east-west direction (not shown in Fig. 11(b)). The resultant magnetic disturbance is in the north-south direction on the ground, i.e., in the $H$ component, because the magnetic field caused by the ionospheric current in the north-south direction is mostly cancelled by the field-aligned currents (Fukushima, 1976). On the other hand, the magnetic field caused by the secondary Hall current is not cancelled and observed on the ground. As seen in Figs. 4, 5, and 8, the tendency that the resonance peaks appear mainly in the $H$ component rather than in the $D$ component is consistent with this expectation.

These qualitative interpretations should be confirmed quantitatively with a three-dimensional realistic numerical simulation of atmospheric gravity waves, including the ionospheric dynamo process, in future, as well as with direct observation by low-altitude magnetic satellites such as CHAMP or SWARM.

Acknowledgments. The geomagnetic data from Memambetsu and Kakioka were provided by the JMA/Kakioka Magnetic Observatory. The barometric data at Mizusawa were provided by the NAOJ/Mizusawa VLBI Observatory. Barometric observations at
Shigaraki are maintained by the RISH/Shigaraki MU Radar Site, Kyoto University. This study was partly supported by the 2011 Kyoto University "Core Stage Backup" program and the $2012 \mathrm{Ky}-$ oto University "Zengaku Keihi” program.

\section{References}

Choosakul, N., A. Saito, T. Iyemori, and M. Hashizume, Excitation of 4-min periodic ionospheric variations following the great Sumatra-Andaman earthquake in 2004, J. Geophys. Res., 114, A10313, doi:10.1029/2008JA013915, 2009.

Fujita, S. and T. Tamao, Duct propagation of hydromagnetic waves in the upper ionosphere, 1, Electromagnetic field disturbances in high latitudes associated with localized incidence of a shear Alfvén wave, J. Geophys. Res., 93, 14,665-14,673, doi:10.1029/JA093iA12p1466591,1988.

Fukushima, N., Generalized theorem for no ground magnetic effect of vertical currents connected with Pedersen currents in the uniformconductivity ionosphere, Rep. Ionos. Space Res. Jpn., 30, 35-50, 1976.

Iyemori, T., M. Nosé, D.-S. Han, Y. Gao, M. Hashizume, N. Choosakul, H. Shinagawa, Y. Tanaka, M. Utsugi, A. Saito, H. McCreadie, Y. Odagi, and F. Yang, Geomagnetic pulsations caused by the Sumatra earthquake on December 26, 2004, Geophys. Res. Lett., 32, L20807, doi:10.1029/2005GL024083, 2005.

Kanamori, H. and J. Mori, Harmonic excitation of mantle Rayleigh waves by the 1991 eruption of Mount Pinatubo, Philippines, Geophys. Res. Lett., 19, 721-724, 1992.

Kanamori, H., J. Mori, and D. G. Harkrider, Excitation of atmospheric oscillations by volcanic eruptions, J. Geophys. Res., 99, 21,947-21,961, 1994.

Lognonne, P., E. Clevede, and H. Kanamori, Computation of seismograms and atmospheric oscillations by normal-mode summation for a spherical earth model with realistic atmosphere, Geophys. J. Int., 135, 388-406, 1998.

Matsumura, M., A. Saito, T. Iyemori, H. Shinagawa, T. Tsugawa, Y. Otsuka, M. Nishioka, and C. H. Chen, Numerical simulations of atmospheric waves excited by the 2011 off the Pacific coast of Tohoku Earthquake, Earth Planets Space, 63, 885-889, 2011.

Nishida, K., N. Kobayashi, and Y. Fukao, Resonant oscillations between the solid Earth and the atmosphere, Science, 287, 2244-2246, 2000.

Saito, A., T. Tsugawa, Y. Otsuka, M. Nishioka, T. Iyemori, M. Matsumura, S. Saito, C. H. Chen, Y. Goi, and N. Choosakul, Acoustic resonance and plasma depletion detected by GPS total electron content observation after the 2011 off the Pacific coast of Tohoku Earthquake, Earth Planets Space, 63, 863-867, 2011.

Selby, N. D., E. Eshun, H. J. Patton, and A.Douglas, Unusual longperiod Rayleigh wave radiation from a vertical dip-slip source: The 7 May 2001 North Sea earthquake, J. Geophys. Res., 110, B10304, doi:10.1029/2005JB003721, 2005.

Shinagawa, H., T. Iyemori, S. Saito, and T. Maruyama, A numerical simulation of ionospheric and atmospheric variations associated with the Sumatora earthquake on December 26, 2004, Earth Planets Space, 59, 1015-1026, 2007.

Tahira, M., Acoustic resonance of the atmosphere at $3.7 \mathrm{mHz}, J$. Atmos. Sci., 52, 2670-2674, 1995.

Tamao, T., Direct contribution of oblique field-aligned currents to ground magnetic fields, J. Geophys. Res., 91, 183-189, doi:10.1029/JA091iA01p00183, 1986.

Tsugawa, T., A. Saito, Y. Otsuka, M. Nishioka, T. Maruyama, H. Kato, T. Nagatsuma, and K. T. Murata, Ionospheric disturbances detected by GPS total electron content observation after the 2011 off the Pacific coast of Tohoku Earthquake, Earth Planets Space, 63, 875-879, 2011.

T. Iyemori (e-mail: iyemori@kugi.kyoto-u.ac.jp), Y. Tanaka, Y. Odagi, Y. Sano, M. Takeda, M. Nose, M. Utsugi, D. Rosales, E. Choque, J. Ishitsuka, S. Yamanaka, K. Nakanishi, M. Matsumura, and H. Shinagawa 